\title{
AGING
}

\section{Quickening the clock for aging studies}

Schultz, M.B. et al. Nat Commun 11, 4618 (2020)

It takes a mouse a long time to grow old. Relatively speaking, of course - the lifespan of a typical laboratory mouse is only about three years, compared to the 80 or so a human can expect. But you'll nevertheless have to wait to see whether an intervention given to an animal while it is young will have any meaningful effect on its life- or health-span as it ages. When the goal is to find a therapeutic that can help prevent or delay the development of an age-related pathology, negative or inconclusive results can mean time and mice wasted. What if instead researchers could speed up the clock?

Two new 'clocks' - models called the Frailty Inferred Geriatric Health Timeline (FRIGHT) and Analysis of Frailty and Death (AFRAID) - won't make your mice age faster, but they may help you predict outcomes and decide whether to continue on earlier in the course of an aging study.

"What we were hoping to do was figure out a way, earlier in the process, of understanding whether an intervention was going to affect lifespan," says Alice Kane, a postdoctoral fellow in David Sinclair's lab at Harvard Medical School. "We were also hoping to provide a standardized health assessment to get information about how healthy the mice are," she says.

The two clocks are based on the Mouse Clinical Frailty Index, a non-invasive health scoring approach developed by Kane's previous postdoctoral advisor, Susan Howlett at Dalhousie University. Reverse translated from the literature on frailty indices in people, the mouse version is calculated from 31 different measures of animal health, including body condition, gait and grip strength, and hearing and eye sight. "It's really a great tool because it has the clinical relevance," says Kane, and it can be performed non-invasively and without any special equipment.

In prior applications, the frailty index score has been shown to increase with age and correlate with a number of health outcomes in mice, says Kane. "But what we really wanted to understand was whether we could use these items to predict outcomes." The lab first needed a complete dataset. "It's kind of unusual for people to track health in mice and then also allow them to naturally die," she says. So over the course of 21

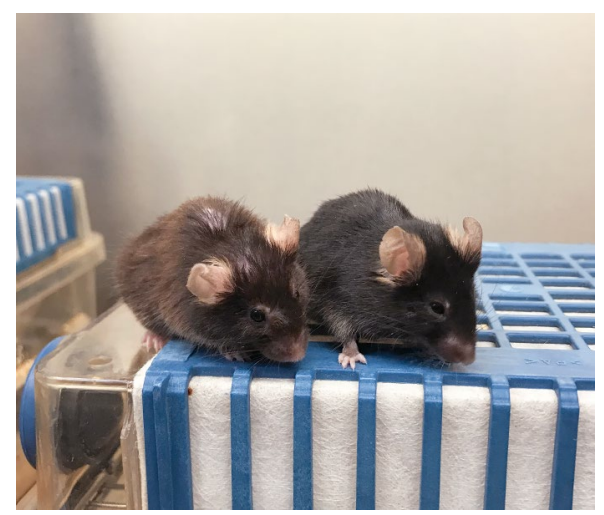

An aged mouse next to a younger companion. Credit: Alice Kane

months, she scored sixty 20 -month old male C57BL/6Nia mice every six weeks through the end of their lives (only four had to be euthanized).

Maximum survival was 36 months of age, and the median, 31 . Despite being from an inbred strain, individual mice did vary in their frailty trajectories as they aged. With the completed lifespan data in hand, she and co-first author Michael Schultz trained a random forest regression, a modeling method that takes into account interactions between different variables, some of which can correlate with an outcome more strongly than others, on the recorded frailty index items to generate the two clocks. For FRIGHT, the largest contributors to the model ended up being breathing rate, tail stiffening, kyphosis (curvature of the spine), and total weight change; the most important variables to AFRAID included total weight loss, actual chronological age, and tremor.

The FRIGHT clock is intended to reveal an animal's apparent chronological age the age it appears, rather than what the calendar says. Like humans, some mice can seem younger than their age suggests, while others may wear their months more heavily. Knowing an animal's apparent age can inform whether an intervention is affecting overall health, says Kane. The FRIGHT clock, however, did not end up being a good predictor of mortality. To address the issue of lifespan, Kane and Schultz then added chronological age to the frailty index items to their random forest modeling to generate the AFRAID clock, which can predict how much longer a mouse is likely to survive from a given time point.

With clocks ticking, Kane and her colleagues then tested their models against two datasets: that from a prior study of enalapril, an Angiotensin-converting enzyme (ACE) inhibitor shown to improve health but not longevity in mice, and new data from mice treated with methionine restriction, a dietary approach that can extend both health- and life- spans. At 27 months, FRIGHT indicated that enalapril-treated mice appeared about a month younger than controls, while the AFRAID prediction concurred that there would be no lengthening of overall lifespan; the clocks agreed with methionine restriction observations as well.

As the underlying data are the same but each analysis can reveal slightly different information, Kane suggests interested researchers apply the Frailty Index as well as both clocks to their own aging cohorts; index score cards can be uploaded at http:// frailtyclocks.sinclairlab.org/ for those who want to run their own results through the FRIGHT and AFRAID models. Meanwhile, follow up with a cohort of female mice which arrived in Boston at 12 months of age from the National Institute of Aging - is underway to assess whether the clocks will work as-is in both sexes, or if female-specific versions will need to be trained; similar considerations might also apply to different mouse strains.

Kane and her colleagues are also exploring whether more refined clocks, incorporating different molecular measures of aging, can be developed as well. With different measures accounted for - some that may be important earlier in life, others later - Kane hopes the clocks themselves will help scientists better understand the aging process itself, in addition to informing whether different interventions may prove potent against age-related declines. "I envision us having levels of clocks," Kane says, "Depending on the lab and your facility and what you can do."

\section{Ellen P. Neff}

Published online: 12 October 2020

https://doi.org/10.1038/s41684-020-00668-w 